\title{
Cómo revelan las sociedades anónimas abiertas chilenas los impuestos diferidos en sus estados financieros ${ }^{1}$
}

\author{
Tania K. López Balmaceda ${ }^{2}$
}

Fabián H. Soto Guzmán ${ }^{3}$

Recibido: 19 de junio de 2019

Aprobado: 30 de julio de 2019

\section{Clasificación JEL: M41}

\section{Resumen}

La globalización, junto con el deseo de crecer que poseen las empresas, representa un gran reto para la contabilidad. Lo anterior genera una gran variedad de transacciones que derivan en una mayor cantidad de información contable y que necesitan contar con nuevos parámetros que controlen su revelación. Las Normas Internacionales de Contabilidad (NIC) solucionan dicho problema y logran un lenguaje común en los negocios, pero ¿̇las compañías cumplen con todos los estándares descritos?

El propósito de esta investigación es observar y analizar cómo revelan la información contable sobre impuestos diferidos las sociedades anónimas abiertas (SAA) chilenas en los estados financieros (EE.FF.), reglamentados

1 López Balmaceda, T. K., \& Soto Guzmán, F. H. (2019). Cómo revelan las sociedades anónimas abiertas chilenas los impuestos diferidos en sus estados financieros. Revista Activos, 17(1), 115-138. DOI: https://doi.org/10.15332/25005278.5396

2 Estudiante de Contabilidad y Auditoría de la Universidad de Santiago de Chile. Santiago de Chile, Chile. Correo electrónico: tania.lopez.b@usach.cl.

3 Estudiante de Contabilidad y Auditoría de la Universidad de Santiago de Chile. Santiago de Chile, Chile. Correo electrónico: fabian.soto.g@usach.cl 
por la NIC 12 "Impuesto a las ganancias". Específicamente los objetivos son: examinar los criterios contables expuestos en la norma, comparar dichos criterios con la información presentada por las entidades en los EE.FF. y establecer características generales que posean las empresas.

Al finalizar el estudio se espera encontrar una aplicación incompleta de la NIC 12 en los estados financieros y una disparidad entre la información revelada por las compañías, en consecuencia de que la norma no especifica una estructura concreta de cómo se debe presentar la información, evidenciando la práctica de comunicar el contenido mínimo exigido sin validar su utilidad.

Palabras clave: impuestos diferidos, NIC 12 "Impuesto a las ganancias", aplicación de normativas en SAA chilenas, revelación de impuestos diferidos.

\title{
How Chilean public limited companies disclose deferred taxes in their financial statements
}

\begin{abstract}
Globalization, together with the desire of companies to grow, represent a great challenge for accounting. This generates a great variety of transactions that result in a greater amount of accounting information that needs to have new parameters that control its disclosure. The International Accounting Standards (IAS) solve this problem, achieving a common language in business, but do companies comply with all the standards described?

The purpose of this research is to observe and analyze how Chilean open stock corporations (SAA) disclose accounting information on deferred taxes in the Financial Statements (EE.FF.), regulated by IAS 12 "Income Taxes". The specific objectives are to examine the accounting criteria set out in the standard, to compare these criteria with the information presented by the entities in the EE.FF. and to establish general characteristics of the companies.
\end{abstract}


At the end of the study it is expected to find an incomplete application of IAS 12 in the Financial Statements, also a disparity between the information disclosed by companies, consequently, that the standard does not specify a specific structure of how the information should be presented, evidencing the practice of communicating the minimum required content without validating its usefulness.

Keywords: Deferred taxes, IAS 12 earnings tax, Application of regulations in Chilean SAA, Revelation of deferred taxes.

\section{Introducción}

Las Sociedades Anónimas Abiertas (SAA) chilenas son las únicas, desde el punto coercitivo, obligadas a llevar su contabilidad aplicando las Normas Internacionales de Información Financiera (NIIF), sin hacer caso omiso a la norma tributaria. Ambas normativas presentan un objetivo propio, las NIIF entregan requisitos sobre la información por revelar de transacciones y hechos económicos que afectan a una empresa, mientras que la norma tributaria destaca principalmente el pago de los tributos al Estado.

La aplicación de ambas normas genera diferencias en la valorización de activos y pasivos, además de consecuencias futuras, y es el objetivo central de la NIC 12 "Impuesto a las ganancias". Dada esta situación es muy importante observar y analizar su correcta aplicación.

Los objetivos son:

- Examinar en profundidad el marco teórico central por utilizar (NIC 12) obteniendo criterios contables.

- Comparar dichos criterios con la información presentada por las entidades en los EE.FF.

- Establecer características generales que posean las empresas. 


\section{Metodología}

Este trabajo se estructura según la metodología planteada por S. J. Taylor y R. Bogdan en su artículo científico "Introducción a los métodos cualitativos" en el que señalan una metodología cualitativa enfocada en analizar un fenómeno a través de una serie de características.

La metodología antes señalada consta de cinco grandes supuestos:

- Es inductiva. Se desarrollan conceptos, intelecciones y comprensiones partiendo de pautas de los datos, y no recogiendo datos para evaluar modelos, hipótesis o teorías preconcebidos.

- El investigador ve al escenario y a las personas en una perspectiva holística; las personas, los escenarios o los grupos no son reducidos a variables, sino considerados como un todo.

- Los investigadores cualitativos son sensibles a los efectos que ellos mismos causan sobre las personas que son objeto de su estudio.

- Los investigadores cualitativos tratan de comprender a las personas dentro del marco de referencia de ellas mismas.

- Para el investigador cualitativo, todas las perspectivas son valiosas.

(Taylor y Bogdan, 2000)

Complementando el enfoque cualitativo, se utilizó un análisis estadístico con la finalidad de observar y analizar cómo revelan la información contable sobre los impuestos diferidos las SAA, teniendo como parámetros los señalados por la NIC 12 "Impuesto a las ganancias". 


\section{Desarrollo de la investigación}

\section{Primera etapa: exploración y análisis del marco teórico}

\subsection{NIC 12 "Impuesto a las ganancias"}

El objetivo de la norma es prescribir el tratamiento contable del impuesto a las ganancias. Uno de los principales problemas que poseen las empresas es la contabilización de las consecuencias actuales y futuras del importe en libros de activos y pasivos, además de distintas transacciones relacionadas. La expectativa de recuperar un activo o liquidar un pasivo está siempre latente, debido a que existe estrecha relación con el pago de impuestos y efectos fiscales.

La norma define tres conceptos relevantes:

Las diferencias temporarias: son las que existen entre el importe en libros de un activo o pasivo en el estado de situación financiera y su base fiscal.

Las diferencias temporarias pueden ser:

a. Diferencias temporarias imponibles.

b. Diferencias temporarias deducibles.

Pasivos por impuestos diferidos: son las cantidades de impuestos sobre las ganancias por pagar en periodos futuros, relacionadas con las diferencias temporarias imponibles.

Activos por impuestos diferidos: son las cantidades de impuestos sobre las ganancias por recuperar en periodos futuros, asociadas con:

a. Las diferencias temporarias deducibles;

b. la compensación de pérdidas obtenidas en periodos anteriores que todavía no hayan sido objeto de deducción fiscal; 
c. la compensación de créditos no utilizados procedentes de periodos anteriores.

(International Accounting Standards Board, 2001)

En resumen, es posible señalar que los pasivos y activos diferidos se generan a partir de diferencias entre el valor según sus bases financieras tributarias. Es importante indicar que dichas diferencias son temporarias y no permanentes, debido a que la legislación da la posibilidad de recuperar o liquidar el importe en un futuro, ya que forman parte de la determinación del gasto tributario por impuesto a la renta y en el correspondiente pasivo por pagar de un ejercicio diferente.

\section{Reconocimiento de pasivos por impuestos diferidos}

La NIC 12 explica que se reconocerá un pasivo por impuestos diferidos por causa de cualquier diferencia imponible que se haya generado, a menos que haya sido por:

a. El reconocimiento inicial de una plusvalía comprada; o

b. el reconocimiento inicial de un activo o pasivo en una transacción que:

i. No es una combinación de negocios, y

ii. en el momento en que fue realizada no afectó ni a la ganancia contable ni a la ganancia (pérdida) fiscal.

(International Accounting Standards Board, 2001)

Asimismo, establece el reconocimiento de un pasivo cuando el importe en libros de un activo supere el valor fiscal de este, dado que la diferencia será imponible. En otras palabras, existe una obligación de pagar los impuestos correspondientes en el futuro. 


\section{Reconocimiento de activos por impuestos diferidos}

La NIC 12 indica que se reconocerá un activo por impuestos diferidos, siempre que haya diferencias temporarias deducibles, dando como resultado posibles ganancias fiscales futuras. Sin embargo, existen dos excepciones a la regla:

a. "No sea una combinación de negocios, y que

b. en el momento de realizarla, no haya afectado ni a la ganancia contable ni a la ganancia (pérdida) fiscal" (International Accounting Standards Board, 2001).

\section{Medición}

Los activos y pasivos por impuestos diferidos deben ser medidos utilizando las tasas fiscales que se aplican en el periodo en que el activo o pasivo se realice. En Chile, la tasa por utilizar depende del tipo de contribuyente; en el caso de las SAA, tributan según el régimen semiintegrado estipulado en el artículo 14 letra B de la Ley de Impuesto a la Renta; por tanto, la tasa por aplicar en 2017 es de un $25.5 \%$.

Asimismo, la norma señala que los activos y pasivos por impuestos diferidos deben ser remedidos al cierre de cada ejercicio, utilizando la tasa fiscal en el momento que se reverse. La diferencia entre el importe inicial y el recalculado se debe contabilizar como cargo o abono a los resultados del ejercicio, a menos que dicha diferencia se relaciona con partidas de patrimonio, siendo esta contabilizada en otros resultados integrales.

\section{Presentación}

La normativa no hace referencia explícita de cómo se debe presentar la información sobre impuestos diferidos en el estado financiero, creando así diferencias y dificultades para comparar la información; lo que sí señala es qué debe ser presentado. 
Un tema relevante por tratar es la compensación entre activos y pasivos diferidos, lo cual solo puede efectuarse si:

Posee el derecho legal para compensar los importes de dichas partidas y

deriva del impuesto a las ganancias correspondiente a la autoridad fiscal.

La NIC 12 hace referencia al gasto o ingreso por impuestos, relacionado con el resultado de las actividades ordinarias, el cual deberá presentarse en el estado del resultado integral. Existe la opción de presentar los componentes en un estado de resultados separado, lo cual se encuentra normado en la NIC 1 "Presentación de estados financieros".

\subsection{Normativa tributaria en Chile}

En Chile los impuestos diferidos también se encuentran normados con el boletín n. 60 Contabilización del impuesto a la renta e impuestos diferidos, el cual complementa a la norma contable antes descrita. Dicha publicación señala estándares para la medición, presentación y revelación de los impuestos, pero con un énfasis ligado a la estimación futura de los efectos tributarios. Al final, el boletín menciona revelaciones en los estados financieros, específicamente lo que debe presentarse en las notas explicativas, en especial fiscales y más generales, y no señala tantos estándares como la NIC 12.

Es relevante señalar que en Chile el Oficio Circular 856 dispuso una excepción a lo normado por la NIC 12: "Las diferencias en activos y pasivos por concepto de impuestos diferidos que se produzcan como efecto directo del incremento de la tasa de impuestos de primera categoría introducido por la Ley 20780, deberán contabilizarse en el ejercicio respectivo contra patrimonio".

En resumen, se genera una distorsión en cuanto a qué información debe revelar la entidad en sus estados financieros. 


\section{Segunda etapa: parámetros descritos}

Estos consisten principalmente en las características que se estudiarán y serán objeto de análisis. Se establecen tres secciones: en la primera se muestra la información general que se espera extraerá de la memoria, la segunda es la información revelada en la nota de las principales políticas contables de la sociedad y la última es la información presentada en la nota de impuestos diferidos.

\section{Sección 1. Aspectos generales}

En esta se indica el rubro al cual pertenece cada sociedad, el número de páginas de la memoria, de los EE.FF. y de la nota específica de impuestos diferidos, para examinar si existe una homogeneidad entre la cantidad de información que pueda presentar cada sociedad.

\section{Sección 2. Nota principales políticas contables}

Pone énfasis en tres puntos principales:

- Nombrar de forma directa la norma que explica el tratamiento de los impuestos diferidos, entendiéndose que esto le resulta de utilidad a los usuarios de la información.

- Detallar de forma clara los criterios que utiliza para el tratamiento de impuestos diferidos y que estos sean iguales a los que indica la norma.

- Mencionar si utiliza el método del balance, el cual consiste en comparar el valor libro contable con el valor tributario (base fiscal).

\section{Sección 3. Nota específica de impuestos diferidos}

En este apartado se han utilizado criterios que se consideran relevantes para la investigación y además se incorpora la información por revelar que señala la NIC 12. Los apartados desarrollados son: los montos de comparación entre lo señalado por los estados financieros y los que presenta la nota explicativa de cada empresa, tanto de activos y pasivos, como de 
impuestos diferidos y el resultado por impuesto a las ganancias; indicar la tasa impositiva por utilizar para el cálculo de impuestos y el desglose de los componentes de activos y pasivos por impuestos diferidos. Con respecto a los datos señalados por NIC 12, se han utilizado la compensación de activos y pasivos por impuestos diferidos (párrafo 74), desglose de componentes de gastos e ingreso por impuesto a las ganancias (párrafo 79), en su mayoría el párrafo 81 a partir de la letra c en adelante y finalmente el párrafo 88 .

\section{Tercera etapa: muestra de empresas por utilizar}

La investigación fue elaborada con una muestra de 171 Sociedades Anónimas Abiertas chilenas (51 \% de la población total), las cuales fueron seleccionadas al azar del listado publicado por la Comisión para el Mercado Financiero, el 9 de julio de 2018. Las entidades fiscalizadas estaban vigentes a la fecha y presentaban su memoria de 2017 publicada.

Con el fin de realizar una representación fidedigna, útil y comparativa, el grupo decidió agrupar la muestra en 17 rubros principales creados mediante los códigos de actividades económicas entregados por el Servicio de Impuestos Internos.

\section{Cuarta etapa: criterios utilizados para el análisis de la información}

Para realizar el análisis se crearon distintas posibles respuestas, las cuales son las siguientes y tienen estas interpretaciones:

Sí: indica que la información es válida y está cumpliendo con lo señalado por la norma, específicamente con los parámetros descritos en la Etapa 2.

No: la información revelada no cumple con los estándares solicitados por la normativa o cumple de manera errónea lo requerido.

No posee: esta categoría, a diferencia del no, es utilizada para indicar que no se presentó la nota explicativa en el estado financiero o no posee impuestos diferidos en ese periodo. 
Se eligieron estas interpretaciones con el fin de simplificar y ayudar a la comprensión de los resultados.

\section{Resultados}

Con el fin de revelar la información obtenida de manera sencilla y fácil de comprender, se ha decidido realizar una simbología con respecto a los rubros, simplificando así sus nombres.

Tabla 1. Rubros de las empresas

\begin{tabular}{l|c}
\hline Rubros & Grupos \\
\hline Actividades artísticas, de entretenimiento y recreativas & Grupo 1 \\
\hline Actividades de atención de la salud humana y de asistencia social & Grupo 2 \\
\hline Actividades de servicios administrativos y de apoyo & Grupo 3 \\
\hline Actividades financieras y de seguros & Grupo 4 \\
\hline Actividades inmobiliarias & Grupo 5 \\
\hline Actividades profesionales, científicas y técnicas & Grupo 6 \\
\hline Agricultura, ganadería, silvicultura y pesca & Grupo 7 \\
\hline $\begin{array}{l}\text { Comercio al por mayor y al por menor; reparación de vehículos } \\
\text { automotores y motocicletas }\end{array}$ & Grupo 8 \\
\hline Construcción & Grupo 9 \\
\hline Enseñanza & Grupo 10 \\
\hline Explotación de minas y canteras & Grupo 11 \\
\hline Industria manufacturera & Grupo 12 \\
\hline Información y comunicaciones & Grupo 13 \\
\hline Otras actividades de servicios & Grupo 14 \\
\hline $\begin{array}{l}\text { Suministro de agua; evacuación de aguas residuales, gestión de } \\
\text { desechos y descontaminación }\end{array}$ & Grupo 15 \\
\hline Suministro de electricidad, gas, vapor y aire acondicionado & Grupo 16 \\
\hline Transporte y almacenamiento & Grupo 17 \\
\hline
\end{tabular}

Nota: rubros según su actividad económica (elaborada por el organismo regulador en temas de tributación interna en Chile, Servicio de Impuestos Internos - SII).

Fuente: elaboración propia. 


\section{Sección 1. Aspectos generales}

A continuación, se presenta un gráfico que señala los aspectos generales. En este se presenta el porcentaje de páginas de los EE.FF. respecto del número total de páginas de la memoria; además, se muestra el porcentaje de páginas de la nota de impuestos diferidos respecto del número de páginas de los EE.FF.

Figura 1. Aspectos generales de la investigación

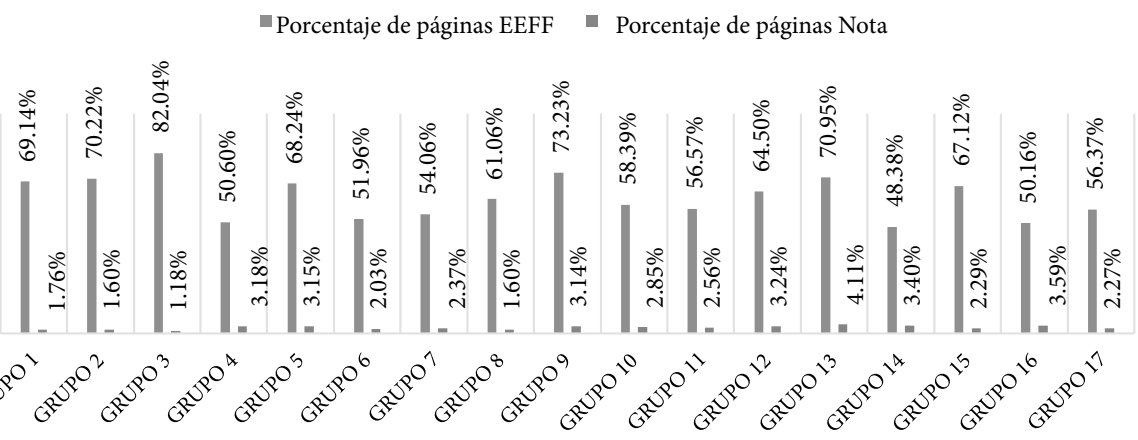

Fuente: elaboración propia.

\section{Sección 2. Nota "Principales políticas contables"}

En el siguiente gráfico se presentan los resultados obtenidos en la Sección 2 referida a la información sobre la nota "Principales políticas contables", la cual normalmente corresponde a la nota 2. Se evaluaron tres puntos relevantes:

- La nota indica el nombre de la NIC estudiada.

- Detalla la NIC 12 en profundidad, lo cual demuestra un manejo contable financiero y tributario.

- Señala el método del balance, el cual derogó al método de resultado. El nuevo método permite reconocer efectos diferidos aun cuando la reversión final sea lejana y destaca que todos los activos y pasivos tendrán efectos en el futuro. 
Figura 2. Nota de principales políticas contables aplicadas

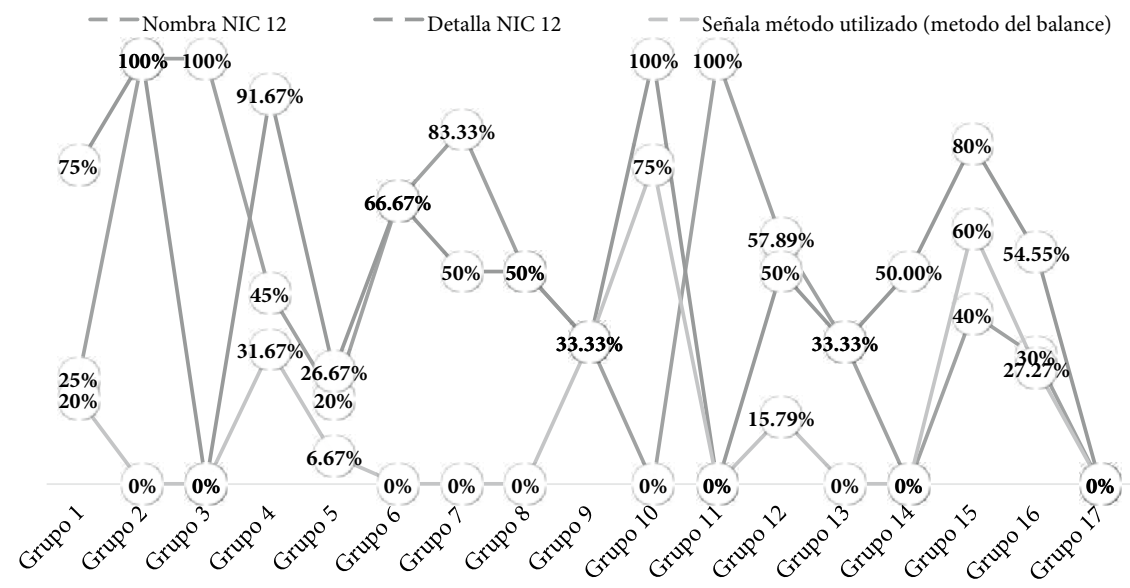

Fuente: elaboración propia.

\section{Sección 3. Nota específica de impuestos diferidos}

Los montos por el resultado por impuesto a las ganancias, activos y pasivos por impuestos diferidos fueron comparados desde el balance general, estado de resultados y la nota explicativa sobre impuestos diferidos, los cuales arrojaron los siguientes datos:

Tabla 2. Comparación de montos entre EE.FF. y notas explicativas

\begin{tabular}{c|c|c|c|c}
\hline Grupo & Concepto & Activo \% & Pasivo \% & Resultado \\
\hline \multirow{3}{*}{ Grupo 1 } & Sí & 45 & 40 & 40 \\
& No & 5 & 10 & 10 \\
& No posee & 50 & 50 & 50 \\
\hline \multirow{3}{*}{ Grupo 2 } & Sí & 100 & 100 & 100 \\
& No & 0 & 0 & 0 \\
& No posee & 0 & 0 & 0 \\
\hline \multirow{3}{*}{ Grupo 3 } & Sí & 100 & 100 & 100 \\
& No & 0 & 0 & 0 \\
& No posee & 0 & 0 & 0 \\
\hline
\end{tabular}




\begin{tabular}{|c|c|c|c|c|}
\hline Grupo & Concepto & Activo \% & Pasivo \% & Resultado \\
\hline Grupo 4 & $\begin{array}{c}\text { Sí } \\
\text { No } \\
\text { No posee }\end{array}$ & $\begin{array}{c}95 \\
3.33 \\
1.67 \\
\end{array}$ & $\begin{array}{c}95 \\
3.33 \\
1.67 \\
\end{array}$ & $\begin{array}{c}96.67 \\
1.67 \\
1.66 \\
\end{array}$ \\
\hline Grupo 5 & $\begin{array}{c}\text { Sí } \\
\text { No } \\
\text { No posee }\end{array}$ & $\begin{array}{c}20 \\
33.33 \\
46.67 \\
\end{array}$ & $\begin{array}{l}26.67 \\
26.66 \\
46.67 \\
\end{array}$ & $\begin{array}{c}33.33 \\
20 \\
46.67 \\
\end{array}$ \\
\hline Grupo 6 & $\begin{array}{c}\text { Sí } \\
\text { No } \\
\text { No posee }\end{array}$ & $\begin{array}{c}100 \\
0 \\
0\end{array}$ & $\begin{array}{c}100 \\
0 \\
0\end{array}$ & $\begin{array}{c}100 \\
0 \\
0\end{array}$ \\
\hline Grupo 7 & $\begin{array}{c}\text { Sí } \\
\text { No } \\
\text { No posee }\end{array}$ & $\begin{array}{c}66.67 \\
33.33 \\
0\end{array}$ & $\begin{array}{c}83.33 \\
16.67 \\
0\end{array}$ & $\begin{array}{c}100 \\
0 \\
0\end{array}$ \\
\hline Grupo 8 & $\begin{array}{c}\text { Sí } \\
\text { No } \\
\text { No posee }\end{array}$ & $\begin{array}{c}83.33 \\
0 \\
16.67\end{array}$ & $\begin{array}{c}83.33 \\
0 \\
16.678 \\
\end{array}$ & $\begin{array}{c}83.33 \\
0 \\
16.67\end{array}$ \\
\hline Grupo 9 & $\begin{array}{c}\text { Sí } \\
\text { No } \\
\text { No posee }\end{array}$ & $\begin{array}{c}100 \\
0 \\
0 \\
\end{array}$ & $\begin{array}{c}100 \\
0 \\
0\end{array}$ & $\begin{array}{c}100 \\
0 \\
0\end{array}$ \\
\hline Grupo 10 & $\begin{array}{c}\text { Sí } \\
\text { No } \\
\text { No posee }\end{array}$ & $\begin{array}{c}100 \\
0 \\
0 \\
\end{array}$ & $\begin{array}{c}100 \\
0 \\
0 \\
\end{array}$ & $\begin{array}{c}75 \\
25 \\
0\end{array}$ \\
\hline Grupo 11 & $\begin{array}{c}\text { Sí } \\
\text { No } \\
\text { No posee }\end{array}$ & $\begin{array}{c}100 \\
0 \\
0\end{array}$ & $\begin{array}{c}100 \\
0 \\
0\end{array}$ & $\begin{array}{c}100 \\
0 \\
0\end{array}$ \\
\hline Grupo 12 & $\begin{array}{c}\text { Sí } \\
\text { No } \\
\text { No posee }\end{array}$ & $\begin{array}{c}94.74 \\
0 \\
5.26\end{array}$ & $\begin{array}{c}94.74 \\
0 \\
5.26\end{array}$ & $\begin{array}{c}94.74 \\
0 \\
5.26\end{array}$ \\
\hline Grupo 13 & $\begin{array}{c}\text { Sí } \\
\text { No } \\
\text { No posee }\end{array}$ & $\begin{array}{c}100 \\
0 \\
0\end{array}$ & $\begin{array}{c}100 \\
0 \\
0\end{array}$ & $\begin{array}{c}100 \\
0 \\
0\end{array}$ \\
\hline Grupo 14 & $\begin{array}{c}\text { Sí } \\
\text { No } \\
\text { No posee }\end{array}$ & $\begin{array}{c}50 \\
0 \\
50\end{array}$ & $\begin{array}{c}50 \\
0 \\
50 \\
\end{array}$ & $\begin{array}{c}50 \\
0 \\
50 \\
\end{array}$ \\
\hline Grupo 15 & $\begin{array}{c}\text { Sí } \\
\text { No } \\
\text { No posee }\end{array}$ & $\begin{array}{c}100 \\
0 \\
0\end{array}$ & $\begin{array}{c}100 \\
0 \\
0\end{array}$ & $\begin{array}{c}100 \\
0 \\
0\end{array}$ \\
\hline
\end{tabular}




\begin{tabular}{c|c|c|c|c}
\hline Grupo & Concepto & Activo \% & Pasivo \% & Resultado \\
\hline \multirow{3}{*}{ Grupo 16 } & Sí & 81.82 & 81.82 & 81.82 \\
& No & 0 & 0 & 0 \\
& No posee & 18.18 & 18.18 & 18.18 \\
\hline \multirow{3}{*}{ Grupo 17 } & Sí & 71.43 & 71.43 & 71.43 \\
& No & 0 & 0 & 0 \\
& No posee & 28.57 & 28.57 & 28.57 \\
\hline
\end{tabular}

Nota: observación de posibles diferencias en montos entre EE.FF. y notas explicativas. Fuente: elaboración propia.

En general la mayor parte de las empresas presentó de forma correcta la información sin provocar contradicciones; por otro lado, las que no poseían la nota o no presentaron impuestos diferidos causaron una leve distorsión de la información. Ahora, aquellas en las cuales no coincidieron los montos, principalmente se caracterizaban por revelar de forma desorganizada la información.

\section{Señala la tasa impositiva por utilizar}

Resulta de mucha utilidad para saber cómo se llegó a determinados valores, además de que la legislación tributaria en Chile se modificó en el último tiempo causando un cambio en las tasas, de forma anual. De la muestra seleccionada se revela que un $72.51 \%$ indica la tasa del periodo, un $12.87 \%$ no indica cuál es la tasa y un $14.62 \%$ no presentó la correspondiente nota.

\section{Desglosa componentes de activo (pasivo) por impuestos diferidos}

Mostrar los componentes del activo y pasivo sirve para explicar el origen de estos y comprender alguna transacción que pueda ser de utilidad para los usuarios de la información; de lo anterior, un $80.12 \%$ de las sociedades anónimas estudiadas desglosan los componentes, mientras que un $5.26 \%$ no lo hace y un $14.62 \%$ no tuvo impuestos diferidos en el periodo o no creó la respectiva nota. 


\section{Párrafo 74}

Tal párrafo describe la compensación de activos y pasivos por impuestos diferidos, además de los requisitos para poder realizarla. Lo que debe presentar una empresa para explicar la compensación es un cuadro que detalle los activos y pasivos que se compensaron. (La norma no lo señala, pero todas las compañías lo revelaron de esa manera).

El 45 \% de las empresas realizó una tabla que explicaba la compensación efectuada.

El 40 \% no explicó el procedimiento o el cumplimento de los requisitos.

Finalmente, el $15 \%$ no presentó la nota explicativa o los impuestos diferidos en el periodo.

\section{Párrafo 79}

Señala los principales elementos que componen al gasto e ingreso por impuesto a las ganancias, los cuales deben revelarse de forma separada. Lo anterior fue aplicado a la muestra y dio como resultados:

Un $79.53 \%$ presenta de forma correcta lo indicado por la norma.

Un $5.85 \%$ no cumple con los estándares solicitados.

Finalmente, un $14.62 \%$ no presentó la nota explicativa o no presentó activos por impuestos diferidos en el periodo.

\section{Párrafo 81, letra a)}

Se exige la revelación del importe de los impuestos, corrientes y diferidos, relacionados directamente con patrimonio, además de presentar el importe relativo a cada componente. 
Lo anterior fue aplicado a la muestra y resultó que en promedio:

Un $70 \%$ de las empresas no poseen impuestos relacionados al patrimonio.

Un $18 \%$ de las compañías señalan que sí poseen impuestos relativos a partidas cargadas al patrimonio neto, de las cuales el $100 \%$ detalla en un cuadro específico cada cuenta afectada.

Un $12 \%$ no posee impuestos diferidos en el periodo o no posee nota específica.

$\mathrm{Al}$ analizar los resultados, se observa un correcto cumplimiento de la norma, dado que el total de las empresas reveló lo solicitado.

\section{Párrafo 81, letra c)}

La NIC 12 indica que se debe revelar una explicación de la relación entre el gasto (ingreso) por el impuesto y la ganancia contable, junto con esto delimita dos posibles maneras de revelar dicha información. ${ }^{4}$

Una conciliación numérica entre el gasto (ingreso) por el impuesto y el resultado de multiplicar la ganancia contable por la tasa impositiva aplicable.

Una conciliación numérica entre la tasa media efectiva y la tasa impositiva aplicable.

Al evaluar dicho estándar en la muestra, los resultados son:

4 La empresa puede utilizar ambas o solo una forma, pero debe especificar la manera de computar la tasa que usó. 
Tabla 3. Relación gasto (ingreso) por impuesto y ganancia contable

\begin{tabular}{l|c|c|c|c|c}
\hline Grupos & $\begin{array}{c}\text { Conciliación } \\
\text { numérica } \\
\%\end{array}$ & $\begin{array}{c}\text { Conciliación } \\
\text { de tasas } \\
\%\end{array}$ & $\begin{array}{c}\text { Ambas } \\
\text { conciliaciones } \\
\%\end{array}$ & $\begin{array}{c}\text { No muestra } \\
\text { explicación } \\
\%\end{array}$ & $\begin{array}{c}\text { No } \\
\text { posee } \\
\%\end{array}$ \\
\hline Grupo 1 & 10 & 20 & 0 & 20 & 50 \\
\hline Grupo 2 & 0 & 100 & 0 & 0 & 0 \\
\hline Grupo 3 & 0 & 100 & 0 & 0 & 0 \\
\hline Grupo 4 & 5 & 57 & 10 & 27 & 2 \\
\hline Grupo 5 & 0 & 20 & 0 & 20 & 60 \\
\hline Grupo 6 & 0 & 100 & 0 & 0 & 0 \\
\hline Grupo 7 & 67 & 33 & 0 & 0 & 0 \\
\hline Grupo 8 & 67 & 17 & 0 & 0 & 17 \\
\hline Grupo 9 & 67 & 0 & 33 & 0 & 0 \\
\hline Grupo 10 & 25 & 0 & 0 & 75 & 0 \\
\hline Grupo 11 & 100 & 0 & 0 & 0 & 0 \\
\hline Grupo 12 & 53 & 0 & 42 & 0 & 5 \\
\hline Grupo 13 & 100 & 0 & 0 & 0 & 0 \\
\hline Grupo 14 & 50 & 0 & 0 & 0 & 50 \\
\hline Grupo 15 & 40 & 0 & 60 & 0 & 0 \\
\hline Grupo 16 & 36 & 0 & 45 & 0 & 18 \\
\hline Grupo 17 & 43 & 0 & 14 & 14 & 29 \\
\hline
\end{tabular}

Nota: conciliación entre gasto e ingreso según lo indicado por las NIIF. Fuente: elaboración propia.

Basándose en los resultados señalados en la tabla 3, es posible observar diferencias en la presentación de la información según cada rubro, pero similitudes entre las empresas pertenecientes al mismo rubro. Es importante destacar que la norma presenta ejemplos que indican cómo realizar las conciliaciones, lo cual facilita a los usuarios la aplicación de este requerimiento.

Gran parte de las compañías prefiere revelar la información utilizando solo una forma para conciliar el gasto (ingreso) por el impuesto y la ganancia contable, y la más empleada es la conciliación numérica. 


\section{Párrafo 81, letra d)}

Las tasas fiscales cambian con mucha frecuencia, por esta razón la NIC 12 solicita una explicación de los cambios en la tasa impositiva aplicable, al menos para exponer la tasa anteriormente utilizada. El estudio reveló que un $47 \%$ de las SAA cumple con este estándar, mientras que un $40 \%$ no lo hace y un $13 \%$ no posee nota explicativa respecto a los impuestos diferidos.

El bajo porcentaje se debe a que la normativa no es clara al momento de cómo revelar la información; si bien muchas empresas describieron el cambio de tasa, no todas lo realizaron en la nota específica sobre impuestos diferidos, sino que aclararon la medición en la nota de "principales criterios contables aplicados".

\section{Párrafo 81, letra e)}

La norma establece que las entidades deben revelar "el importe (y fecha de validez, si la tuvieran), de las diferencias temporarias deducibles, pérdidas o créditos fiscales no utilizados para los cuales no se hayan reconocido activos por impuestos diferidos en el estado de situación financiera" (International Accounting Standards Board, 2001).

Tomando como referencia lo descrito, se aplica dicho parámetro a la muestra, lo cual da como resultado que en promedio un $9 \%$ de las SAA sí presentaban el importe y la fecha de validación, un $78 \%$ no presenta diferencias temporarias deducibles, pérdidas o créditos fiscales no utilizados y un $13 \%$ no posee nota respectiva o no posee impuestos diferidos.

En resumen, las entidades que muestran diferencias temporarias no utilizadas cumplen, en su totalidad, con la normativa. 
Figura 3. Porcentaje de diferencias temporarias relacionadas con inversiones

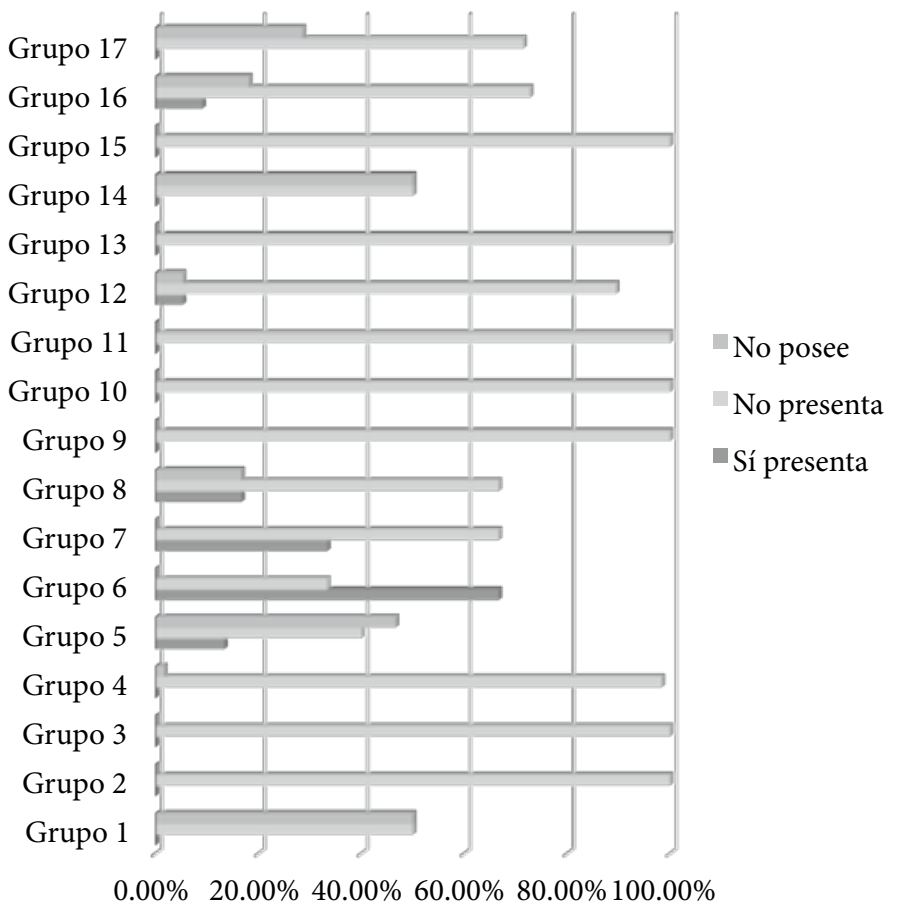

Fuente: elaboración propia.

\section{Párrafo 81, letras f) y g)}

La letra f) menciona que las empresas deben revelar "la cantidad total de diferencias temporarias relacionadas con inversiones en subsidiarias, sucursales y asociadas, o con participaciones en negocios conjuntos, para los cuales no se han reconocido pasivos por impuestos diferidos" (International Accounting Standards Board, 2001).

Con base en la figura 3 es posible percibir que la mayor parte de las empresas no presenta diferencias temporarias con inversiones, llegando así a un $79 \%$. Por otra parte, un $8 \%$ sí presenta y menciona la cantidad 
total de diferencias temporarias y un $13 \%$ no posee nota explicativa o no tiene impuestos diferidos.

Así también, la NIC 12 indica que deben ser reconocidos el importe de los activos y pasivos por impuestos diferidos que fueron reconocidos en el estado de situación financiera y los que fueron reconocidos en el resultado del periodo. Al analizar la muestra, se pudo apreciar que el $100 \%$ de las entidades que presentaron diferencias temporarias y pérdidas o créditos fiscales no utilizados revelaron el importe en el estado de situación financiera, mientras que solo un $88 \%$ presentaron el importe en el resultado del periodo.

En resumen, la norma fue aplicada de una manera incompleta, sin cumplir con la totalidad de parámetros solicitados y exigidos.

\section{Párrafo 81, letras h) e i)}

Respecto a las operaciones discontinuadas, la norma hace referencia a estas y exige revelar el gasto por impuestos relativo a:

(i) "La ganancia o pérdida derivada de la discontinuación, y

(ii) la ganancia o pérdida del periodo por las actividades ordinarias de la operación discontinuada, junto con los importes correspondientes para cada uno de los periodos anteriores presentados" (International Accounting Standards Board, 2001).

$\mathrm{Al}$ analizar este criterio en la muestra, se pudo observar que ninguna empresa reveló el gasto por impuestos, sino que el $87 \%$ no presentó operaciones discontinuadas y el $13 \%$ restante no presentó nota explicativa o no posee impuestos diferidos. Igual ocurre con la letra i) del mismo párrafo: en el importe de las consecuencias en el impuesto sobre las ganancias de los dividendos para los accionistas de la entidad, el resultado es idéntico.

En resumen, 149 empresas no revelaron cambios en el gasto por impuesto relativo a operaciones discontinuadas ni a dividendos para los 
accionistas. Esto puede deberse a que es un parámetro muy específico y no ocurre con tanta regularidad.

\section{Párrafo 81, letras j) y k)}

La NIC 12 expone el tratamiento y la revelación de impuestos diferidos por combinación de negocios. Específicamente destaca dos puntos:

Señalar el importe reconocido del activo por impuestos diferidos del adquiriente, si cambia antes de la adquisición.

Reconocer si los beneficios por impuestos diferidos adquiridos en una combinación de negocios no están reconocidos en la fecha de la adquisición, pero lo hayan sido tras dicha fecha.

Luego de aplicar este estándar a la muestra, fue posible observar que el $4 \%$ de las empresas reveló la letra a) y un $1 \%$ reveló la b); eso quiere decir que siete compañías habrían reconocido un cambio en el importe de activo por impuesto diferido.

Cabe destacar que en promedio el $85 \%$ de la muestra no reveló ninguno de los puntos, debido a que no poseen cambios en los activos por impuestos diferidos.

\section{Párrafo 88}

Como último parámetro de la investigación se decidió examinar si las entidades revelaban información acerca de pasivos y activos contingentes que tengan relación con los impuestos. Pero a la fecha, ninguna de las 171 empresas presentó información acerca de lo antes mencionado. Un 87 \% no mostró activos y pasivos contingentes, mientras que el $13 \%$ restante no presentó nota explicativa o no posee impuesto diferido. 


\section{Conclusiones}

Al finalizar el estudio, puede apreciarse que existe disparidad entre las compañías, específicamente diferencias entre los grupos. Las empresas que se encontraban en el mismo rubro evidenciaban poseer un patrón al revelar la información; esto se debe principalmente al vacío que poseen las Normas Internacionales de Contabilidad, las cuales no delimitan un formato para la información por revelar. La NIC 12 cumple la función de exigir contenido, pero no valida la utilidad de dicha información provocando que el concepto de comparabilidad entre los estados financieros sea más difícil de realizar.

Asimismo, cabe señalar que la norma no fue correctamente aplicada por las compañías, teniendo errores que demostraban un mal manejo de conceptos. El incluir lenguaje fiscal y financiero genera una norma más compleja en relación con las otras.

Para concluir esta investigación se responderá la pregunta central: ¿Cómo revelan las Sociedades Anónimas Abiertas chilenas los impuestos diferidos en sus estados financieros? La manera en que dichas entidades presentan la información es con base en las Normas Internacionales de Contabilidad y la legislación chilena (Ley de Impuesto a la Renta, Oficio Circular 856, entre otras) cumpliendo con los parámetros correspondientes. Aunque existan empresas que no cumplan con la totalidad, la mayoría de las sociedades mostraba lo que se le solicitaba de manera clara.

Se quiere postular una solución a los problemas mencionados, con el fin de que esta investigación no termine y siga desarrollándose. La propuesta es generar una norma que delimite la presentación en los estados financieros, con el fin de mejorar la comprensión y comparabilidad entre entidades en el ámbito general. 


\section{Referencias}

Deloitte. (2017). Guía rápida de las NIIF. Recuperado de https://www2.deloitte. com/ni/es/pages/audit/articles/niif-bolsillo.html

Fowler Newton, E. (2006). Normas Internacionales de Información Financiera. Buenos Aires: La Ley.

International Accounting Standards Board. (2001). NIC 12 "Impuesto a las ganancias".

Pérez, V. y Pinto Perry, G. (2013). Alcances de la aplicación de la NIC 12 en Argentina y Chile. Cofin Habana, revista cubana de contabilidad y finanzas, (1), enero-marzo, 48-56. Recuperado de http://www.cofinhab.uh.cu/index. $\mathrm{php} / \mathrm{RCCF} / \mathrm{article} / \mathrm{view} / 90 / 89$

Ruz Farías, V. (2014). Una doctrina sobre las IFRS (2. ${ }^{a}$ edición). Santiago de Chile: Smart Ediciones.

Servicio de Impuestos Internos - SII. (s. f.). Boletín técnico $n .^{\circ}$ 60. Contabilización del impuesto a la renta e impuestos diferidos. Recuperado de http://www.sii. $\mathrm{cl} /$ pagina/actualizada/contadores/boletin60.htm

SVS - Superintendencia de Valores y Seguros. (2014). Oficio Circular $n .^{\circ} 856$. Santiago de Chile. Recuperado de http://www.cmfchile.cl/normativa/ ofc_856_2014.pdf

Taylor, S. J. y Bogdan, R. (2000). Introducción a los métodos cualitativos de investigación. Buenos Aires: Paidós. Recuperado de https://iessb.files.wordpress. com/2015/07/05_taylor_mc3a9todos.pdf

Zgaib, A. (2004). El impuesto diferido. Conceptos básicos, aspectos controvertidos y casos prácticos (2. ${ }^{\text {a } e d i c i o ́ n) . ~ B u e n o s ~ A i r e s: ~ L a ~ L e y . ~}$ 